\title{
Beijing Declaration of International Association of Neurorestoratology (IANR) \\ - Version Beijing 2009 -
}

\author{
Adopted by the 2nd IANR Annual Conference General Assembly, Beijing, China, April 26, 2009
}

1. With rapid development of basic and clinical advancement on neural restoration, neurorestoratology has become a distinct discipline within neurosciences.

2. World wide neurological impairment due to neurodegeneration and neural injury is a major factor reducing human longevity and quality of life. Neurorestoratology seeks therapeutic solutions to this problem.

3. Neurorestoratology is the sub-discipline of neuroscience that studies neural regeneration, repair and replacement of damaged components of the nervous system, neuroplasticity, neuroprotection and neuromodulatory mechanisms of recovery. The goal of neurorestoratology is to promote recovery of neural function.

4. The research and scope of neurorestoratology cover causes and treatments of neurotrauma, neurodegeneration, cerebrovascular anoxia or ischemia, edema, demyelination, sensory and motor disorders, and neuropathic pain, as well as neural damage resulting from toxic, physical and chemical factors, immune, infectious, inflammatory, hereditary, congenital, developmental, and other causes of neural injury.

5. Neurorestorative therapies include regeneration and reconstruction of nervous tissues by transplantation of tissue, cells, or biomaterials and bioengineering, modulation by electro-magnetic stimulation, pharmaceutical or chemical therapies, and combinations of the above.

6. Functional recovery is the objective of neurorestoratology, requiring close integration of neurorestorative and neurorehabilitative therapies, as well as the practice of clinical and preventative medicine to minimize complications of neural degeneration and damage.

7. Neurorestoratology recognizes the importance of small functional gains that have significant effects on quality of life. Neurorestoratology is interested in the mechanisms of spontaneous activity and enhancing this recovery.

8. Neurorestoratology supports the highest standards for clinical trials to evaluate the safety and efficacy of its neurorestorative therapies, the establishment of validated outcome measures, and ethical treatment of patients.

9. The International Association for Neurorestoratology will develop guidelines for neurorestorative therapies, seek and encourage development of new therapies, promote multi-center collaboration, and will contribute to the training of people in the field.

Proposed by: Hong-Yun HUANG (China), Geoffrey Raisman (UK), Wagih S El Masri (UK), Da-Jue WANG (UK), Tian-Sheng SUN (China), En-Zhong LIU (China), Huan-Cong ZUO (China), Chang-Man ZHOU (China), Lin CHEN (China), Michael Chopp (USA), Almudena Ramon-Cueto (Spain), Albert Bohbot (France), Ziad .M. Al Zoubi (Jordan), Klaus R.H. von Wild (Germany), Robert M. Levy (USA), Eva Sykova (Czech), Carlos Lima (Portugal), Patricia E. Phelps (USA), Gerson Chadi (Brazil), Chee-Pin CHEE (Malaysia), Elena Chernykh (Russia), Debora R. Fior Chadi (Brazil), Krystyna Domanska-Janik(Poland), Foo-Chiang LEE (Malaysia), Gustavo Moviglia (Argentina), Yoon Ha (Korea), Russell J Andrews (USA), Milan Dimitrijevic (USA \& Slovenia), Paul R. Sanberg (USA), Mrabet. Abdelfattah (Tunis), Jian-Xing SONG (Singapore), Wise Young (USA). 